\title{
EDITORIAL
}

For reprint orders, please contact: reprints@futuremedicine.com

\section{Taking radiation out of the equation in primary mediastinal B-cell lymphoma}



\author{
"...we found that DA-EPOCH-R is a highly \\ curable strategy in primary mediastinal B-cell \\ lymphoma that, importantly, removes the \\ need for mediastinal radiation in almost \\ all cases."
}

Clifton Mo ${ }^{1}$, Cliona Grant ${ }^{2}$, Mark Roschewski ${ }^{1,2}$ \& Kieron Dunleavy*2

Primary mediastinal B-cell lymphoma (PMBL) is a subtype of diffuse large $B$-cell lymphoma (DLBCL) that arises in the thymus [1]. PMBL accounts for up to $10 \%$ of cases of DLBCL and predominantly affects females in the third and fourth decade of life. Its clinical presentation is distinct from that of other types of DLBCL and resembles nodular sclerosing Hodgkin lymphoma in that it typically presents with an anterior mediastinal mass that may infiltrate into adjacent structures, such as the lungs and chest wall [1]. Its molecular profile is also unique and much closer to nodular sclerosing Hodgkin lymphoma (they share approximately a third of their genes) than to the other subtypes of DLBCL [2].

Among other reasons, the rarity of this entity and the paucity of prospective studies have resulted in a lack of consensus on the optimal therapeutic approach and choice of regimen for PMBL [3]. Early studies in PMBL suggested that mediastinal radiation was a necessary component of curative therapy and most strategies today continue to incorporate it. One of the early studies that led to this practice evaluated the methotrexate, leucovorin, doxorubicin, cyclophosphamide, vincristine, prednisone and bleomycin (MACOP-B) regimen followed by consolidative radiation treatment. While only $19 \%$ of patients had a positive gallium scan at the completion of radiotherapy, $66 \%$ were gallium positive after chemotherapy alone and this supported a combined modality approach (following which $80 \%$ were event-free at 39 months median follow-up time) [4]. The need for regimens with increased dose intensity has been another controversial topic. As dose intensity is important in the treatment of Hodgkin lymphoma, it would not be unexpected for this to be the case in PMBL, given their shared clinical and biological characteristics [5]. Several retrospective studies have explored this question [6-8]. In the first of these by Todeschini et al., MACOP-B and etoposide, doxorubicin, cyclophosphamide, vincristine, prednisone and bleomycin (VACOP-B) were compared with cyclophosphamide, doxorubicin, vincristine and prednisone (CHOP). The outcome for patients in the latter group was inferior suggesting a role for dose intensity [6].

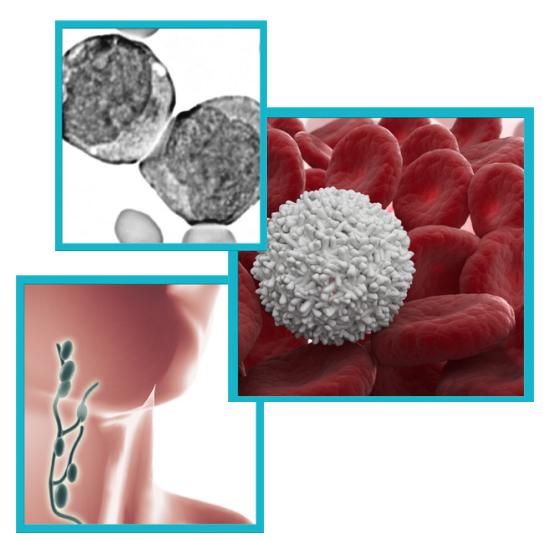

"Among other reasons, the rarity of this entity and the paucity of prospective studies have resulted in a lack of consensus on the optimal therapeutic approach and choice of regimen for primary mediastinal B-cell lymphoma."

'Walter Reed National Military Medical Center, 8901 Wisconsin Avenue, Bethesda, MD 20814, USA

${ }^{2}$ Metabolism Branch, National Cancer Institute, Building 10, Room 4N/115, 9000 Rockville Pike, Bethesda, MD 20892, USA *Author for correspondence: dunleavk@mail.nih.gov

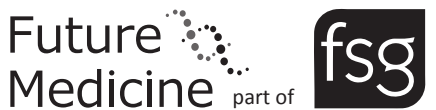


A second study conducted by the International Extranodal Lymphoma Study Group (IELSG) compared the outcomes of $426 \mathrm{PMBL}$ patients who received MACOP-B, VACOP-B, cyclophosphamide, doxorubicin, etoposide cytozar, bleomycin, vincristine, methotrexate and prednisone (ProMACECytaBom) or CHOP and again, the outcome in the CHOP arm was inferior [7]. A third retrospective study by the Memorial Sloan-Kettering Cancer Center group compared dose-dense approaches to CHOP-like therapy in PMBL and concluded that dose-dense chemotherapy may be superior to CHOP [8]. However, there have not been prospective comparisons of dose-intense versus conventional approaches in PMBL specifically. At the time when the Southwest Oncology Group (SWOG) prospectively compared CHOP to second- and thirdgeneration regimens in DLBCL, PMBL was not recognized as a distinct entity and the outcome of patients treated with first- versus second- or third-generation regimens was not assessed [9].

"Although high cure rates can be
achieved in primary mediastinal B-cell
lymphoma following combined
modality treatment, it is clear ... that
the use of mediastinal radiation is
associated with significant and
devastating late-term sequelae, such
as secondary tumors and cardiac
disease."

While the addition of rituximab to $\mathrm{CHOP}$ chemotherapy (R-CHOP) in DLBCL has been shown to improve survival in several different studies, it has not been well studied in PMBL, again due to the rarity of the disease. In a retrospective study conducted in British Columbia (Canada) in the pre- and post-rituximab eras, there was no survival advantage seen when rituximab was added to $\mathrm{CHOP}$ (however, the number of patients in the R-CHOP arm was small, and the follow-up time was relatively short) [10]. Other studies, meanwhile, have suggested a benefit to adding rituximab. In the randomized, Phase III MabThera International Trial (MInT), a subgroup analysis looked at the additive role of rituximab in combination with CHOP-like regimens in PMBL [11]. The rituximab arm was clearly superior in terms of 3 -year event-free survival (78 vs $52 \%$ in the chemotherapy arm alone), but no statistically significant difference in overall survival was detected due to small numbers. More recently, studies by Vissilakopolous et al. [12] and Soumerai et al. [13] reported 5-year event-free and progression-free survivals of 80 and $68 \%$ in 76 and 58 patients treated with R-CHOP with or without radiation $[12,13]$. Of importance in interpreting all of these results is the fact that preplanned radiotherapy was administered to the majority of patients who received $\mathrm{R}-\mathrm{CHOP}$ and the addition of radiation improved remission rates.

"Based on these results, and to provide confirmatory evidence of this strategy in primary mediastinal B-cell lymphoma, an international trial of DA-EPOCH-R is ongoing in children with primary mediastinal B-cell lymphoma."

Although high cure rates can be achieved in PMBL following combined modality treatment, it is clear, particularly from our collective experience in Hodgkin lymphoma, that the use of mediastinal radiation is associated with significant and devastating late-term sequelae, such as secondary tumors and cardiac disease $[14,15]$. The excess risk of breast cancer is particularly problematic given that the majority of patients with PMBL are young women. Lower doses of radiation may reduce the risk of these complications but this is controversial and, in fact, a recent survivorship study in pediatric Hodgkin lymphoma demonstrated that secondary tumors occurred with similar latency and frequency in patients who received low-dose versus high-dose radiation [16].

Due to the aforementioned evidence suggesting that dose intensity is likely to be an important component of optimal PMBL therapy, we investigated the dose-adjusted etoposide, prednisone, vincristine, cyclophosphamide, doxorubicin and rituximab (DA-EPOCH-R) regimen in this disease, based on its efficacy in DLBCL $[17]$. This is a pharmacodynamically dose-adjusted regimen and this strategy may especially benefit younger patients who likely require higher doses of drugs to achieve similar serum drug levels to older patients [18]. We set out to investigate if this regimen could obviate the need for mediastinal radiation and thus, eliminate the risk of its long-term sequelae. We treated 51 patients, most of whom were female, with a median age of 30 years. The majority 
presented with bulky mediastinal disease and an elevated lactate dehydrogenase level. At a median follow-up of 63 months, the event-free and overall survival were 93 and $97 \%$, respectively [19]. Only three patients failed to achieve a complete response (two had evidence of persistent focal disease on FDG-PET CT and one had progressive disease); two of these patients went on to receive mediastinal radiotherapy and one was observed after excisional biopsy. To date, no patients have relapsed. Additionally, in order to obtain an independent assessment of DA-EPOCH-R in PMBL we collaborated with investigators at Stanford University (CA, USA) who treated 16 consecutively newly diagnosed patients and at a median follow-up of 37 months, all patients were alive and without disease and, importantly, none had received radiotherapy. In terms of assessing the additive benefit of rituximab with this regimen, when these results were compared with a historical PMBL group who received DA-EPOCH alone (no radiation), the addition of rituximab significantly improved event-free and overall survival [19]. The toxicity of DA-EPOCH-R was similar to previous reports and importantly, we found that the infusional schedule of doxorubicin allowed for the delivery of high cumulative doses of doxorubicin without any significant cardiac toxicity.

In conclusion, we found that DA-EPOCH-R is a highly curable strategy in PMBL that, importantly, removes the need for mediastinal radiation in almost all cases. Given the young median age of patients with this disease, eliminating the long-term risks of radiation toxicity while maintaining high cure rates is an important therapeutic advancement. Based on these results, and to provide confirmatory evidence of this strategy in PMBL, an international trial of DA-EPOCH-R is ongoing in children with PMBL (ClinicalTrials.gov number, NCT01516567 [101]).

\section{Financial \& competing interests disclosure}

The authors have no relevant affiliations or financial involvement with any organization or entity with a financial interest in or financial conflict with the subject matter or materials discussed in the manuscript. This includes employment, consultancies, honoraria, stock ownership or options, expert testimony, grants or patents received or pending, or royalties.

No writing assistance was utilized in the production of this manuscript.

\section{References}

1 Swerdlow SH, Campo E, Harris NL et al. WHO Classification of Tumours of Haematopoietic and Lymphoid Tissues. IARC, Lyon, France (2008).

2 Rosenwald A, Wright G, Leroy $\mathrm{K}$ et al. Molecular diagnosis of primary mediastinal B cell lymphoma identifies a clinically favorable subgroup of diffuse large B cell lymphoma related to Hodgkin lymphoma. J. Exp. Med. 198, 851-862 (2003).

3 Dunleavy K, Grant C, Eberle FC, Pittaluga S, Jaffe ES, Wilson WH. Gray zone lymphoma: better treated like hodgkin lymphoma or mediastinal large B-cell lymphoma? Curr. Hematol. Malig. Rep. 7 , 241-247 (2012).

4 Zinzani PL, Martelli M, Magagnoli M et al. Treatment and clinical management of primary mediastinal large B-cell lymphoma with sclerosis: MACOP-B regimen and mediastinal radiotherapy monitored by (67) gallium scan in 50 patients. Blood 94 , 3289-3293 (1999).

5 Diehl V, Franklin J, Pfreundschuh M et al. Standard and increased-dose BEACOPP chemotherapy compared with COPP-ABVD for advanced Hodgkin's disease. N. Engl. J. Med. 348, 2386-2395 (2003).

6 Todeschini G, Secchi S, Morra E et al. Primary mediastinal large B-cell lymphoma (PMLBCL): long-term results from a retrospective multicentre Italian experience in 138 patients treated with $\mathrm{CHOP}$ or MACOP-B/VACOP-B. Br. J. Cancer 90, 372-376 (2004).

7 Zinzani PL, Martelli M, Bertini M et al. Induction chemotherapy strategies for primary mediastinal large B-cell lymphoma with sclerosis: a retrospective multinational study on 426 previously untreated patients. Haematologica 87, 1258-1264 (2002).

8 Hamlin PA, Portlock CS, Straus DJ et al. Primary mediastinal large B-cell lymphoma: optimal therapy and prognostic factor analysis in 141 consecutive patients treated at Memorial Sloan Kettering from 1980 to 1999. Br. J. Haematol. 130, 691-699 (2005).

9 Fisher RI, Gaynor ER, Dahlberg S et al. Comparison of a standard regimen (CHOP) with three intensive chemotherapy regimens for advanced non-Hodgkin's lymphoma. N. Engl. J. Med. 328, 1002-1006 (1993).
10 Savage KJ, Al-Rajhi N, Voss $\mathrm{N}$ et al. Favorable outcome of primary mediastinal large B-cell lymphoma in a single institution: the British Columbia experience. Ann. Oncol. 17, 123-130 (2006).

11 Rieger M, Osterborg A, Pettengell R et al. Primary mediastinal B-cell lymphoma treated with CHOP-like chemotherapy with or without rituximab: results of the Mabthera International Trial Group study. Ann. Oncol. 22, 664-670 (2011).

12 Vassilakopoulos TP, Pangalis GA, Katsigiannis A et al. Rituximab, cyclophosphamide, doxorubicin, vincristine, and prednisone with or without radiotherapy in primary mediastinal large B-cell lymphoma: the emerging standard of care. Oncologist 17, 239-249 (2012).

13 Soumerai JD, Hellmann MD, Feng Y et al. Treatment of primary mediastinal B-cell Lymphoma with R-CHOP is associated with a high rate of primary refractory disease. Leuk. Lymphoma doi:10.3109/10428194. 2013.810738 (2013) (Epub ahead of print).

14 Castellino SM, Geiger AM, Mertens AC et al. Morbidity and mortality in long-term 
EDITORIAL Mo, Grant, Roschewski \& Dunleavy

survivors of Hodgkin lymphoma: a report from the childhood cancer survivor study. Blood 117, 1806-1816 (2011).

15 Dunleavy K, Bollard CM. Sobering realities of surviving Hodgkin lymphoma. Blood 117, 1772-1773 (2011).

16 O'Brien MM, Donaldson SS, Balise RR, Whittemore AS, Link MP. Second malignant neoplasms in survivors of pediatric Hodgkin's lymphoma treated with low-dose radiation and chemotherapy. J. Clin. Oncol. 28, 1232-1239 (2010).
17 Wilson WH, Dunleavy K, Pittaluga S et al. Phase II study of dose-adjusted EPOCH and rituximab in untreated diffuse large B-cell lymphoma with analysis of germinal center and post-germinal center biomarkers. $J$. Clin. Oncol. 26, 2717-2724 (2008).

18 Wilson WH, Porcu P, Hurd D et al. Phase II study of dose-adjusted EPOCH-R in untreated de novo $\mathrm{CD} 20^{+}$diffuse large B-cell lymphoma (DLBCL)-CALGB 50103. J. Clin. Oncol. 16(Suppl.), Abstract 6530 (2005).
19 Dunleavy K, Pittaluga S, Maeda LS et al. Dose-adjusted EPOCH-rituximab therapy in primary mediastinal B-cell lymphoma. N. Engl. J. Med. 368, 1408-1416 (2013).

\section{- Website}

101 Intergroup Trial for Children or Adolescents With Primary Mediastinal Large B-Cell Lymphoma: DA-EPOCH-Rituximab Evaluation.

http://clinicaltrials.gov/show/NCT01516567 